\title{
Global existence and uniqueness for the lake equations with vanishing topography : elliptic estimates for degenerate equations
}

\author{
Didier Bresch *and Guy MÉtivier ${ }^{\dagger}$
}

November 14, 2006

\begin{abstract}
This paper deals with global existence and uniqueness for the lake equations with a bottom topography vanishing on the shore. Our result generalizes previous studies that assumed the depth to be nondegenerate. Elliptic estimates for degenerate equations are established studying the behavior of the associated Green function.
\end{abstract}

Keywords : Regularity result, degenerate elliptic equation, weighted Sobolev spaces, vorticity-Stream function formulation, Youdovitch's method.

AMS subject classification : 35Q30, 35B40, 76D05.

\section{Introduction}

In this paper we are interested in a two-dimensional geophysical model that has been essentially described by Greenspan in $\mathrm{Gr}$ page 235. This system describes the evolution of the vertically averaged horizontal component $v(t, x, y)$ of a three dimensional velocity vector $u(t, x, y, z)$ and reads

$$
\left\{\begin{array}{l}
\partial_{t}(b v)+\operatorname{div}_{\mathbf{x}}(b v \otimes v)+b \nabla_{\mathbf{x}} p=0 \text { in } \Omega, \\
\operatorname{div}_{\mathbf{x}}(b v)=0 \text { in } \Omega, \\
(b v) \cdot n=0 \text { on } \partial \Omega, \\
\left.(b v)\right|_{t=0}=m_{0} \text { in } \Omega
\end{array}\right.
$$

${ }^{*}$ LMC-IMAG, (CNRS-INPG-UJF) 38051 Grenoble cedex, France. Email: didier.bresch@imag.fr

${ }^{\dagger}$ MAB et IUF, Université de Bordeaux I, 33405 Talence cedex, France. Email : Guy.Metivier@math.u-bordeaux.fr 
where $\mathbf{x}$ denotes the horizontal components $\mathbf{x}=(x, y)$. The lake equations may be seen as the low Froude number limit of the usual inviscid shallow water equations when the initial height converges to a nonconstant function depending on the space variable, namely $b(\mathbf{x})$, see for instance BrGiLi]. Recall that the inviscid shallow water reads

$$
\left\{\begin{array}{l}
\partial_{t} h+\operatorname{div}_{\mathbf{x}}(h v)=0 \text { in } \Omega, \\
\partial_{t}(h v)+\operatorname{div}_{\mathbf{x}}(h v \otimes v)+h \nabla_{\mathbf{x}} \frac{(h-b)}{\mathrm{Fr}^{2}}=0 \text { in } \Omega, \\
(h v) \cdot n=0 \text { on } \partial \Omega, \\
\left.(h v)\right|_{t=0}=m_{0},\left.\quad h\right|_{t=0}=h_{0} \text { in } \Omega,
\end{array}\right.
$$

where Fr is the Froude number, $h$ is total depth, $v$ the vertical mean value of the horizontal components of the velocity.

The constant case $b=$ const: The $2 D$ incompressible Euler equations. We remark that the case $b \approx 1$ reduces to the standart 2D incompressible Euler system. The Cauchy problem for incompressible Euler equations is very well understood and we refer the reader to various existing surveys on the question : see for instance $[\mathrm{YO}], \mathrm{Ma}],[\mathrm{Ch}]$ and $[\mathrm{BM}]$. Concerning the bounded domain case, we refer to [Li] where the method used by Youdovitch is explained and various results described. The approach is the construction of the solutions as the inviscid limit of solutions to a Navier-Stokes system with artificial viscosity and boundary conditions $v \cdot n=0$ and $\omega=0$ where $\omega=\partial_{y} v_{1}-\partial_{x} v_{2}$ is the vorticity. We also mentionned [CMR where a wall law related to the cauchy stress tensor replaces the null condition on the vorticity. In all these works, to get the result, elliptic estimates are used on the following equation

$$
-\Delta_{\mathbf{x}} \Psi=\omega \text { in } \Omega,\left.\quad \Psi\right|_{\partial \Omega}=0 .
$$

Namely the following estimate

$$
\|\Psi\|_{W^{2, p}(\Omega)} \leq C p\|\omega\|_{L^{p}(\Omega)}
$$

where $C$ does not depend on $p$. Such elliptic estimates with nondegenerate coefficients follow from AgDoNi1- AgDoNi2]. We also mention in $\mathbb{R}^{2}$ the method based on approximate solutions which strongly uses the following property on $\nabla_{\mathbf{x}} v$, see for instance $\overline{B M}$ : Let $v$ be a smooth, $L^{2}\left(\mathbb{R}^{2}\right) \cap L^{\infty}\left(\mathbb{R}^{2}\right)$, divergence free field and let $\omega=\operatorname{curl} v$, then

$$
\|\nabla v\|_{L^{\infty}\left(\mathbb{R}^{2}\right)} \leq c\left(1+\ln ^{+}\|v\|_{L^{3}\left(\mathbb{R}^{2}\right)}+\ln ^{+}\|\omega\|_{L^{2}\left(\mathbb{R}^{2}\right)}\right)\left(1+\|\omega\|_{L^{\infty}\left(\mathbb{R}^{2}\right)}\right)
$$


where $\ln ^{+} g=0$ if $g \leq 1$ and $\ln ^{+} g=\ln g$ if $g>1$.

The nonconstant case $b \neq$ const: The lake equations. If the depth is not assumed to be constant, we have to define a new vorticity variable to get a transport equation on this quantity. Namely defining

$$
\omega=\frac{1}{b}\left(\partial_{y} v_{1}-\partial_{x} v_{2}\right)
$$

we get that it is transported along the flow $v$ namely

$$
\partial_{t} \omega+v \cdot \nabla_{\mathbf{x}} \omega=0 .
$$

Together with the incompressibility condition $\operatorname{div}(b v)=0$, this implies, formally, conservation of the $L^{p}(b d x)$ norms of $\omega$, for $p \in[1, \infty]$.

The case $b \geq C>0$ has been studied in [LOT proving global existence and uniqueness for an initial vorticity $\omega_{0}$ in $L^{\infty}(\Omega)$ following Youdovitch's method. The regularity of the lake equations has been also addressed in [Ca] in this case but where $|\nabla b(x)| \rightarrow \infty$ as $x$ tends to the boundary. Here we study the degenerate case, namely the case when $b$ stricly positive in $\Omega$ vanishing on the shore $\partial \Omega$. Note that, even if the model seems to be not physically relevant in the presence of beaches, similar models are used by several geophysicits. In a forthcoming paper, the authors will try to better understood the right model to be considered in the presence of beaches.

We will assume that $b>0$ on $\Omega$ and $b=0$ on $\partial \Omega$. More precisely, we assume that $\Omega$ is bounded with smooth boundary $\partial \Omega$, and

$$
b=\varphi^{a}
$$

where $a>0$ and $\Omega=\{\varphi>0\}$ with $\varphi \in C^{\infty}(\bar{\Omega})$ and $\nabla_{\mathbf{x}} \varphi \neq 0$ on $\partial \Omega$.

In order to get our result, an elliptic regularity result is required for a degenerate equation on the associated stream-function. This is the main part of the paper. It is strongly related to a careful study of the associated Green function given in GS-GS1.

The paper is organized as follows. The main results will be summarized in Section 2. In section 3 we will mention the existence and uniqueness result and the key result of the paper: Namely the elliptic estimates on the stream function $\Psi$. Sections 4, 5, 6 are respectively devoted to the proof of such $L^{p}$ estimates by proving the normal regularity and vanishing property, by showing the Hölder estimates and finally by establishing the $L^{p}$ estimates respectively. The well posedness result will be proved in the last section adapting Youdovitch's proof used for the standart 2D incompressible Euler equations.

For the sake of simplicity, throughout the paper, we will supress the $\mathbf{x}$ index from the partial derivatives. 


\section{Main results.}

The goal of the paper is to prove an existence and uniqueness result of a global weak solution for the inviscid equations 1.1. We assume that $\Omega$ is a smooth simply connected bounded domain and that $b$ is given by (1.4). As usual, we eliminate the pressure $p$ from the equation and consider the vorticity-stream formulation of the $2 D$ lake equation. The weak form of the transport equation (1.3) is

$$
\partial_{t}(b \omega)+\operatorname{div}(b \omega v)=0 .
$$

The vorticity and and the velocity are linked by the equations:

$$
\operatorname{div}(b v)=0, \quad \operatorname{curl} v=b \omega, \quad(b v) \cdot n_{\mid \partial \Omega}=0 .
$$

This leads, see for instance [MaPu], to introduce a stream function $\Psi$ such that

$$
v=\frac{1}{b} \nabla^{\perp} \Psi=\frac{1}{b}\left(\partial_{2} \Psi,-\partial_{1} \Psi\right),\left.\quad \Psi\right|_{\partial \Omega}=0 .
$$

In the non-simply connected case, which is discussed later in a remark, one has to specify different boundary values on each connected component of $\partial \Omega$, see $\mathrm{MaPu}$. The model for $\Psi$ then reads

$$
\operatorname{div}\left(\frac{1}{b} \nabla \Psi\right)=b \omega, \quad \Psi_{\mid \partial \Omega}=0 .
$$

Note that, by Hardy's inequalities, the space $C_{0}^{\infty}(\Omega)$ is dense in the space $\left\{\Psi \in H_{0}^{1}(\Omega) ; b^{-1 / 2} \nabla \Psi \in L^{2}(\Omega)\right\}$. Therefore, for $f \in L^{2}(\Omega)$ the problem

$$
\operatorname{div}\left(\frac{1}{b} \nabla \Psi\right)=f, \quad \Psi_{\mid \partial \Omega}=0,
$$

has a unique solution $\Psi$ in this space, denoted by $\Psi=K f$. We make the following definition.

Definition 2.1. Given $\omega_{0} \in L^{\infty}(\Omega),(v, \omega)$ is a weak solution to the vorticitystream formulation of the $2 D$ lake equation with initial data $\omega_{0}$, provided

i) $\left.\omega \in L^{\infty}([0, T]) \times \Omega\right)$ and $b \omega \in C^{0}\left([0, T] ; L_{w *}^{\infty}(\Omega)\right)$,

ii) $b v=\nabla^{\perp} K(b \omega) \in C^{0}\left([0, T] ; L^{2}(\Omega)\right.$,

iii) For all $\varphi \in C^{1}([0, T] \times \bar{\Omega})$ and $t_{1} \in[0, T]$ :

$$
\int_{\Omega} b \varphi\left(t_{1}, x\right) \omega\left(t_{1}, x\right) d x-\int_{\Omega} b \varphi(0, x) \omega_{0}(x) d x=\int_{0}^{t_{1}} \int_{\Omega}\left(b \partial_{t} \varphi+b v \cdot \nabla \varphi\right) \omega d x d t .
$$


Theorem 2.2. i) (Regularity) Assume that $\omega \in L^{\infty}((0, T) \times \Omega)$ and $b v \in L^{\infty}\left([0, T] ; L^{2}(\Omega)\right)$ such that $\operatorname{div}(b v)=0$ satisfy the weak formulation. Then $\omega \in C^{0}\left([0, T] ; L^{r}(\Omega)\right)$ and $v \in C^{0}\left([0, T] ; W^{1, r}(\Omega)\right)$ for all $r<+\infty$. Moreover, there is $C$ such that for all $p \geq 3$,

$$
\|\nabla v\|_{L^{p}(\Omega)} \leq C p\|b \omega\|_{L^{p}(\Omega)} .
$$

In addition, we get the following boundary condition on $v$

$$
v \cdot n=0 \text { on } \partial \Omega .
$$

ii) (Existence) For all $\omega_{0} \in L^{\infty}(\Omega)$, there exists a global weak solution $(v, \omega)$ to the vorticity-stream formulation of (1.1).

iii) (Uniqueness) The weak vorticity-stream solution is unique.

This result follows the Youdovitch's procedure in constructing the solution as the inviscid limit of solutions of a system with artificial viscosity which is the analog of Navier-Stokes with respect to the Euler equations. The core of the proof is a regularity result for a system degenerate due to coefficient vanishing on the boundary of the domain. More precisely, the main part of the paper will concern the following main result. Let $\Omega$ be a smooth simply connected bounded domain in $\mathbb{R}^{n}$ with $n \geq 1$ and $b$ defined by (1.4). Consider the system:

$$
\left\{\begin{array}{l}
\operatorname{div}(b v)=0 \text { in } \Omega, \quad(b v) \cdot n_{\mid \partial \Omega}=0, \\
\operatorname{curl} v=f \text { in } \Omega
\end{array}\right.
$$

for

$$
\left\{\begin{array}{l}
b v \in L^{2}(\Omega), \\
f \in L^{p}(\Omega) .
\end{array}\right.
$$

Using the definition of $b$ given in (1.4), we prove the following result on which the existence and uniqueness result is based.

Theorem 2.3. If $(v, f)$ satisfy (2.6) and (2.7) with $p \in] 2, \infty[$, then

$$
v \in C^{1-n / p}(\bar{\Omega}), \quad \nabla v \in L^{p}(\Omega) .
$$

There is a constant $C_{p}$ independent of $(u, \omega)$ such that

$$
\|v\|_{C^{1-n / p}(\bar{\Omega})} \leq C_{p}\left(\|f\|_{L^{p}}+\|b v\|_{L^{2}}\right) .
$$


In addition

$$
v \cdot n=0 \quad \text { on } \partial \Omega .
$$

Moreover, for all $p_{0}>2$, there is a constant $C$ independent of $(v, f)$ and $p \in\left[p_{0}, \infty[\right.$ such that

$$
\frac{1}{p}\|\nabla v\|_{L^{p}} \leq C\left(\|f\|_{L^{p}}+\|b v\|_{L^{2}}\right) .
$$

In this statement, for $\mu \in] 0,1\left[, C^{\mu}(\bar{\Omega})\right.$ is the usual space of continuous functions on $\bar{\Omega}$ which satisfy the Hölder condition of order $\mu$. In particular, (2.9) implies that

$$
\|v\|_{L^{\infty}(\Omega)} \leq C\left(\|f\|_{L^{\infty}}+\|b v\|_{L^{2}}\right) .
$$

Remark 2.4. It is remarkable that the final $W^{1, p}$ smoothness of $v$ is independent of $b$ in (2.6). When $p=2$, it is still true that $v \in W^{1,2}$ and indeed estimate (2.11) is uniform for $p \in[2, \infty[$. This is shown using estimates of $\mathrm{BG}, \mathrm{BC}$ in the proof below in place of those of [BCM]. However, in this paper we are mainly interested in these estimates as $p \rightarrow \infty$, and we leave aside the case $p=2$. Note also that (2.9) follows from (2.11) and the Sobolev embedding $W^{1, p} \subset C^{\mu}$ for $\mu=1-2 / p>0$. The proof of the uniform estimate (2.11) given below relies on proving (2.9) for a given $p_{0}>n$ first, and on winning the final $L^{p}$ estimate for $\nabla v$, then. This is why we have stated them independently.

Remark 2.5. We include here some remarks on the link between the vorticity-stream formulation and (11.1).

1. Suppose first that $v \in \mathcal{C}^{0}\left([0, T], H^{1}(\Omega)\right)$ is a solution in the sense of distributions of (1.1). For $u \in H^{1}$, the following identity holds:

$$
\operatorname{curl}\left(\frac{1}{b} \operatorname{div}(b u \otimes u)\right)=\operatorname{div}((\operatorname{curl} u) u)-\operatorname{curl}\left(\frac{1}{b}(\operatorname{div} b u) u\right)
$$

implying that $\omega=\operatorname{curl} v$ satisfies transport equation (2.1) in the sense of distributions thus condition iii) in Definition 2.1. Moreover, (2.2) is satisfied by (1.1) and by definition of $\omega$. Thus $v=b^{-1} \nabla^{\perp} \Psi$, (see the beginning of section 3) and the stream function $\Psi \in H_{0}^{1}$ satisfies (2.4), hence $v=$ $b^{-1} \nabla^{\perp} K(b \omega)$ as in $\left.i i\right)$ of Definition 2.1.

2. Conversely, suppose that $(\omega, v)$ is a weak solution of the vorticitystream formulation. By part $i)$ of Theorem $2.2 v \in \mathcal{C}^{0}\left([0, T], L^{2}(\Omega)\right)$ and, 
because $\omega$ satisfies (2.1), we also have $\partial_{t} \omega \in L^{\infty}\left([0, T], H^{-1}(\Omega)\right)$. In particular,

$$
\operatorname{div}\left(b \partial_{t} v\right)=0, \quad \operatorname{curl}\left(\partial_{t} v\right)=b \partial_{t} \omega \in L^{\infty}\left([0, T], H^{-1}(\Omega)\right),
$$

implying that $\partial_{t} v \in L^{\infty}\left([0, T], L_{l o c}^{2}(\Omega)\right)$. The same computation as in 1), implies that in the sense of distributions

$$
\left.\operatorname{curl}\left(\partial_{t} v+\frac{1}{b} \operatorname{div}(b v \otimes v)\right)\right)=\partial_{t}(b \omega)+\operatorname{div}(b v \omega)=0 .
$$

Therefore, assuming that $\Omega$ is simply connected, there is a pressure $p \in$ $L^{\infty}\left([0, T], \mathcal{D}^{\prime}(\Omega)\right)$ such that (1.1) is satisfied.

Remark 2.6. When $\Omega$ has some islands that means it is a non-simply connected domain, some generalized circulations are specified in order to uniquely determine the velocity $v$, see $\mathrm{MaPu}$. More precisely let $\Omega$ with $n$ islands $I_{1}, \ldots, I_{n}$, the missing parameters are the boundary values $\lambda_{i}$ with $i=1, \ldots, n$ of the stream function on each of the boundary component $\partial I_{1}$, $\ldots, \partial I_{n}$. Assuming the generalized circulations to be zero, the velocity is given by $v=v_{0}+\sum_{i=1}^{n} \lambda_{i} v_{i}$ with $v_{j}=\left(\nabla^{\perp} \Psi_{j}\right) / b$ where $\Psi_{j}(j=0, \ldots, n)$ are the unique solution of

$$
-\operatorname{div}\left(\frac{1}{b} \nabla \Psi_{i}\right)=0,\left.\quad \Psi_{i}\right|_{\partial I_{j}}=\delta_{i, j} \quad j=0, \ldots, n \quad i=1, \ldots, n .
$$

and

$$
-\operatorname{div}\left(\frac{1}{b} \nabla \Psi_{0}\right)=b \omega,\left.\quad \Psi_{0}\right|_{\partial \Omega}=0 .
$$

The $n$ coefficients $\lambda_{j}$ are uniquely determined using the zero generalized circulations conditions. This decomposition shows that our study concerning the simply connected domain may be applied to the non-simply connected one, the $L^{p}$ estimate remaining true.

\section{Localization}

Since $b v \in L^{2}(\Omega)$ satisfies $\operatorname{div}(b v)=0$ and $(b v) \cdot n=0$ on $\partial \Omega$, there is a unique potential $\Psi$ such that

$$
v=\frac{1}{b} \nabla^{\perp} \Psi=\frac{1}{b}\left(\partial_{y} \Psi,-\partial_{x} \Psi\right), \quad \Psi_{\mid \partial \Omega}=0 .
$$

Indeed, $\Psi$ is determined in $H_{0}^{1}(\Omega)$ as the solution of

$$
-\Delta \Psi=\operatorname{curl}(b v), \quad \Psi_{\mid \partial \Omega}=0 .
$$


There holds

$$
\operatorname{curl}(b v)=b \operatorname{curl} v+\nabla b \times v=b f-\frac{1}{b} \nabla b \cdot \nabla \Psi
$$

With (1.4), we obtain the equation

$$
-\varphi \Delta \Psi+a \nabla \varphi \cdot \nabla \Psi=\varphi^{a+1} f, \quad \Psi_{\mid \partial \Omega}=0 .
$$

At this stage, the dimension $d=2$ plays no particular rule and we can consider this equation on any smooth bounded domain $\Omega=\{\varphi>0\} \subset \mathbb{R}^{n}$, with $\varphi \in C^{\infty}\left(\mathbb{R}^{n}\right)$ and $d \varphi \neq 0$ on $\partial \Omega$. One introduces the Hölder spaces $C^{\mu}(\bar{\Omega})$ and the Sobolev spaces $W^{k, p}(\Omega)$ based on $L^{p}$, together with the weighted spaces $C_{1}^{\mu}(\bar{\Omega})$ and $W_{1}^{k, p}(\Omega)$ of functions $u$ in $C^{\mu}(\bar{\Omega})$ or $W^{k, p}(\Omega)$ such that $\varphi u$ in $C^{\mu+1}(\bar{\Omega})$ or $W^{k+1, p}(\Omega)$ respectively.

Writing

$$
\Psi=\varphi^{a+1} \Phi, \quad u=\varphi^{-a} \nabla^{\perp} \Psi=\varphi \nabla^{\perp} \Phi+(a+1) \Phi \nabla^{\perp} \varphi,
$$

Theorem 2.3 follows from

Theorem 3.1. If $\Psi \in H_{0}^{1}(\Omega)$ and $f \in L^{p}(\Omega)$ with $p>n$ satisfy (3.3), then $\Phi=\varphi^{-(a+1)} \Psi$ satisfies

$$
\Phi \in \bigcap_{\mu \leq 1-n / p} C_{1}^{\mu}(\bar{\Omega}), \quad \Phi \in \bigcap_{q \leq p} W_{1}^{1, p}(\Omega) .
$$

Moreover, there are constants $C_{\mu}, \mu \in[0,1-n / p]$ such that for all $\Psi \in H_{0}^{1}$ and $f \in L^{p}$ satisfying (3.3),

$$
\|\Phi\|_{C_{1}^{\mu}(\bar{\Omega})} \leq C_{\mu}\left(\|f\|_{L^{p}}+\|\Psi\|_{H^{1}}\right),
$$

For all $p_{0}>n$, there is a constant $C_{1}$, such that for all $\Psi \in H_{0}^{1}$ and $f \in L^{p}$ with $p \geq p_{0}$ satisfying 3.3 )

$$
\frac{1}{p}\|\Phi\|_{W_{1}^{1, p}} \leq C_{1}\left(\|f\|_{L^{p}}+\|\Psi\|_{H^{1}}\right) .
$$

Equation (3.3) is a particular case of degenerate elliptic equations studied in [BG, BG, GS, BCM] and the estimates (3.5) (3.6) are mainly consequences of the results obtained in these papers. Because it may be useful in other circumstances and also because it helps to understand the analysis, we will 
prove the estimates for a slightly more general class of equations, namely equations of the form

$$
L\left(x, \partial_{x}\right) \Psi:=-\varphi(x) P_{2}\left(x, \partial_{x}\right) \Psi+a P_{1}\left(x, \partial_{x}\right) \Psi=\varphi^{a+1} f
$$

where $a$ is a positive real constant and

$$
P_{2}=\sum_{j, k=1}^{n} p_{j, k}(x) \partial_{j} \partial_{k}, \quad P_{1}=\sum_{j=1}^{n} p_{j}(x) \partial_{j}
$$

have real and smooth coefficients on $\bar{\Omega}$, with $p_{j, k}(x)=p_{k, j}(x)$. We further assume that $P_{2}$ is uniformly elliptic on $\bar{\Omega}$

$$
\sum p_{j, k}(x) \xi_{i} \xi_{k}>0 \quad \text { for } x \in \bar{\Omega}, \xi \in \mathbb{R}^{n} \backslash\{0\},
$$

and

$$
P_{1}(x, \xi)=\sum p_{j, k}(x) \xi_{j} \partial_{k} \varphi(x) \quad \text { on } \partial \Omega .
$$

Notice that $(3.9)$ and $(3.10)$ are independent of the choice of the defining function $\varphi$ for $\Omega$.

Theorem 3.2. With assumptions as above, if $\Psi \in H_{0}^{1}(\Omega)$ and $f \in L^{p}(\Omega)$ satisfy (3.7), then $\Phi=\varphi^{-(a+1)} \Psi$ satisfies (3.4) and there are constants $C_{\mu}$ such that the estimates (3.5) and (3.6) hold.

Reduction to a neighborhood of the boundary. Standard elliptic theory implies that on any open subset $\Omega_{1} \subset \Omega, \Psi$ belongs to $W^{2, p}\left(\Omega_{1}\right)$ for all $p$ finite and thus to $C^{\mu+1}\left(\bar{\Omega}_{1}\right)$ for all $\mu \leq 1-n / p$. Moreover,

$$
\|\Phi\|_{C^{\mu+1}\left(\Omega_{1}\right)} \leq C_{\mu}\left(\|f\|_{L^{p}}+\|\Psi\|_{H^{1}}\right)
$$

and, for $p \in[2, \infty[$ :

$$
\frac{1}{p}\left\|\nabla^{2} \Phi\right\|_{L^{p}\left(\Omega_{1}\right)} \leq C_{1}\left(\|f\|_{L^{p}}+\|\Psi\|_{H^{1}}\right)
$$

Therefore, if $\chi \in C^{\infty}(\bar{\Omega})$ is such that $\chi-1 \in C_{0}^{\infty}(\Omega)$, then

$$
L(\chi \Psi)=\varphi^{a+1} \chi f+g \quad \Psi_{\mid \partial \Omega}=0,
$$

where the commutator $g$ is $C^{1-\varepsilon}$ by (3.11) and vanishes on a neighborhood of $\partial \Omega$. Therefore, one can factor out any power of $\varphi$, writing $g=\varphi^{a+1} f_{1}$ with $f_{1} \in L^{\infty}$ with norm bounded by the right hand side of (3.11). Therefore, it is sufficient to prove the estimates (3.5)-(3.6) for functions $\Phi$ that are supported in an arbitrary small neighborhood of $\partial \Omega$. 
Local coordinates near the boundary. The boundary $\partial \Omega$ is a closed smooth manifold. Consider a coordinate patch $x^{\prime} \mapsto \gamma\left(x^{\prime}\right)$ from an open set $\omega \subset$ $\mathbb{R}^{n-1}$ to $\partial \Omega$, with $\gamma \in C^{\infty}$ on $\bar{\omega}$. Taking $\nu\left(x^{\prime}\right)$ to be the inward normal to $\partial \Omega$ at $\gamma\left(x^{\prime}\right)$, we parametrize a neighborhood of $\gamma(\omega)$ by $\left.\left(x^{\prime}, x_{n}\right) \in \omega \times\right]-\delta, \delta[$ by considering the mapping

$$
\Gamma:\left(x^{\prime}, x_{n}\right) \mapsto \gamma\left(x^{\prime}\right)+x_{n} \nu\left(x^{\prime}\right) .
$$

In these coordinates, the equation (3.7) is transformed to

$$
\widetilde{L} \Psi=-x_{n} \widetilde{P}_{2} \Psi+a \widetilde{P}_{1} \Psi=x_{n}^{a+1} f .
$$

The ellipticity property (3.9) is preserved as well as (3.10) which now reads

$$
\widetilde{P}_{1}\left(x^{\prime}, 0, \xi\right)=\sum_{j=1}^{n} \tilde{p}_{j, n}\left(x^{\prime}, 0\right) \xi_{j} .
$$

Collecting all these remarks, we see that Theorem 3.2 follows from the next estimates.

Theorem 3.3. If $\Psi \in H^{1}(\omega \times] 0, \delta[)$ and $f \in L^{p}(\omega \times[0, \delta])$ with $p>n$ satisfy (3.13) and

$$
\Psi_{\mid x=0}=0, \quad \Psi_{\mid\{x>\delta / 2\}}=0 .
$$

then $\Psi=x_{n}^{a+1} \Phi$ with

$$
\Phi \in C_{1}^{1-n / p}\left(\bar{\omega}_{1} \times[0, \delta]\right), \quad \Phi \in W_{1}^{1, p}\left(\omega_{1} \times[0, \delta]\right)
$$

for all relatively compact open subset $\omega_{1} \subset \omega$. Moreover, there are constants $C_{\mu}, \mu \in[0,1-n / p]$ such that for all $\Psi \in H_{0}^{1}$ and $f \in L^{p}, p \geq p_{0}>n$ satisfying (3.3),

$$
\begin{aligned}
\|\Phi\|_{C_{1}^{\mu}\left(\bar{\omega}_{1} \times[0, \delta[)\right.} & \leq C_{\mu}\left(\|f\|_{L^{p}}+\|\Psi\|_{H^{1}}\right), \\
\frac{1}{p}\|\Phi\|_{W_{1}^{1, p}\left(\omega_{1} \times[0, \delta]\right)} & \leq C_{1}\left(\|f\|_{L^{p}}+\|\Psi\|_{H^{1}}\right) .
\end{aligned}
$$

Here $C_{1}^{\mu}\left[\right.$ resp. $\left.W_{1}^{1, p}\right]$ denote the spaces for functions $u \in C^{\mu}[$ resp. $\left.u \in W^{1, p}\right]$ such that $x_{n} u \in C^{\mu+1}\left[\right.$ resp. $x_{n} u \in W^{2, p}$. 


\section{Smoothness in normal direction and vanishing at the boundary}

The first step in the proof of Theorem 3.3 is to factor out $x_{n}^{a+1}$ in $\Psi$ and to show that $\Phi$ is Hölder continuous in the normal variable. For simplicity, we drop the tildes in equation (3.13).

Proposition 4.1. With assumptions as in Theorem 3.3 there are $\mu>0$, $C$ and a Banach space $E \subset \mathcal{D}^{\prime}(\omega)$, such that the function $\Phi=x_{n}^{-(a+1)} \Psi$ satisfies on $\omega \times[0, \delta]$ :

$$
\Phi \in C^{\mu}([0, \delta] ; E), \quad x_{n} \partial_{x_{n}} \Phi \in C^{\mu}([0, \delta] ; E) .
$$

together with the estimates

$$
\|\Phi\|_{C^{\mu}([0, \delta] ; E)}+\left\|x_{n} \partial_{x_{n}} \Phi\right\|_{C^{\mu}([0, \delta] ; E)} \leq C\left(\|f\|_{L^{p}}+\|\Psi\|_{H^{1}}\right) .
$$

Multiplying the equation (3.13) by an appropriate factor, one can always assume that the coefficient of $\partial_{x_{n}}^{2}$ is exactly $x_{n}$. Then, (3.16) implies that the coefficient of $\partial_{x_{n}}$ in $P_{1}$ is 1 when $x_{n}=0$. Thus (3.13) reads

$$
-x_{n} \partial_{x_{n}}^{2} \Psi+a \partial_{x_{n}} \Psi=x_{n}^{a+1} f+F, \quad F=L^{\prime} \Psi
$$

with

$$
L^{\prime}:=\sum_{j, k<n} x_{n} p_{j, k} \partial_{j} \partial_{k}+x_{n} \sum_{j<n} p_{j, n} \partial_{j} \partial_{x_{n}}-a \sum_{j<n} p_{j} \partial_{y}-a \tilde{p}_{n} x_{n} \partial_{x_{n}}
$$

where the $\tilde{p}_{n}$ is smooth.

We consider functions of $x_{n}$ valued in (Banach) spaces of distributions in $x^{\prime}: L^{2}([0, \delta] ; E), C^{\mu}([0, \delta], E)$ etc, with $E \subset \mathcal{D}^{\prime}(\omega)$. The space $E$ is subject to change from line to line, and we note $L^{2}\left(\mathcal{D}_{\mathrm{tg}}^{\prime}\right), C^{\mu}\left(\mathcal{D}_{\mathrm{tg}}^{\prime}\right)$ when the space is unspecified. We also denote by $x_{n}^{\alpha} \mathcal{D}_{\text {tg }}^{\prime}$ the space of products of the function $x_{n}^{\alpha}$ with arbitrary distributions in $\mathcal{D}^{\prime}(\omega)$.

We know that

$$
\Psi \in H^{1} \subset C^{1 / 2}\left(L^{2}\right) .
$$

Using the equation (4.3), we also see that $x_{n} \partial_{x_{n}}^{2} \Psi \in L^{2}\left(H^{-1}\right)$ and hence

$$
x_{n} \partial_{x_{n}} \Psi \in H^{1}\left(H^{-1}\right) \subset C^{1 / 2}\left(H^{-1}\right) .
$$

We will make use of the next (classical) results (See also Proposition 4.1.2 in BCM for more general results). 
Lemma 4.2. For $\alpha>0$ and $\mu \in] 0,1[$, the operators

$$
I_{\alpha} u\left(x_{n}\right)=\int_{0}^{x_{n}}\left(\frac{t}{x_{n}}\right)^{\alpha} u(t) \frac{d t}{t}, \quad J_{\alpha} u\left(x_{n}\right)=\int_{x_{n}}^{\delta}\left(\frac{x_{n}}{t}\right)^{\alpha} u(t) \frac{d t}{t}
$$

map continuously $C^{\mu}([0, \delta] ; E)$ to $C^{\mu}([0, \delta] ; E)$.

Proof of Proposition 4.1. Because $\Psi=0$ for $x \geq \delta / 2$, Equation (4.3) implies that

$$
\partial_{x} \Psi=J_{a}\left(x_{n}^{a+1} f+F\right)=x_{n}^{a} \theta+J_{a}(F)
$$

with

$$
\theta=-\int_{x_{n}}^{\delta} f(t) d t \in C^{1 / 2}\left(L^{p}\right)
$$

Because $\Psi \in H^{1}$ vanishes at $x_{n}=0$, there holds

$$
\Psi=\int_{0}^{x_{n}} \partial_{x_{n}} \Psi(t) d t=x_{n} I_{1}\left(\partial_{x_{n}} \Psi\right) .
$$

a) Suppose that for some $\alpha \in[0, a[$ there is $\mu \in] 0,1[$ such that

$$
\Psi \in x_{n}^{\alpha} C^{\mu}\left(\mathcal{D}_{\mathrm{tg}}^{\prime}\right), \quad x_{n} \partial_{x_{n}} \Psi \in x_{n}^{\alpha} C^{\mu}\left(\mathcal{D}_{\mathrm{tg}}^{\prime}\right)
$$

From (4.5) (4.6), this is true when $\alpha=0$ and $\mu=1 / 2$.

This condition implies that $F=x_{n}^{\alpha} F_{1}$ with $F_{1} \in C^{\mu}\left(\mathcal{D}_{\text {tg }}^{\prime}\right)$. Therefore, $J_{a}(F)=x^{\alpha} J_{a-\alpha}\left(F_{1}\right)$ and (4.8) implies that

$$
\partial_{x_{n}} \Psi=x_{n}^{a} \theta+x_{n}^{\alpha} J_{a-\alpha}\left(F_{1}\right) \in x^{a} C^{1 / 2}\left(\mathcal{D}_{\mathrm{tg}}^{\prime}\right)+x_{n}^{\alpha} C^{\mu}\left(\mathcal{D}_{\mathrm{tg}}^{\prime}\right) \subset x_{n}^{\alpha} C^{\mu_{1}}\left(\mathcal{D}^{\prime}\right)
$$

for some $\mu_{1}>0$. This shows that (4.11) holds with $\alpha$ replaced by $\alpha+1$, decreasing $\mu$ if necessary.

Moreover, if (4.11) holds for some $\alpha$, it also holds for all $\alpha^{\prime}<\alpha$, with possibly smaller $\mu$ 's. Therefore, after a finite number of iterations, one obtains that (4.11) is satisfied for some $\alpha \in] a, a+1[$ and $\mu>0$.

b ) The property (4.11) implies again that $F=x_{n}^{\alpha} F_{1}$ with $F_{1} \in C^{\mu}\left(\mathcal{D}_{\text {tg }}^{\prime}\right)$. We now write

$$
\begin{aligned}
J_{a}(F)= & -x_{n}^{\alpha} I_{\alpha-a}\left(F_{1}\right)+x_{n}^{a} \int_{0}^{\delta} t^{\alpha-a-1} F_{1}(t) d t \\
& \in x_{n}^{\alpha} C^{\mu}\left(\mathcal{D}_{y}^{\prime}\right)+x_{n}^{a} \mathcal{D}_{\mathrm{tg}}^{\prime} \subset x_{n}^{a} C^{\mu_{1}}\left(\mathcal{D}_{y}^{\prime}\right)
\end{aligned}
$$


for some $\mu_{1}>0$. Here we have used that $\alpha>a$.

With (4.8) this implies that $\partial_{x_{n}} \Psi=x_{n}^{a} \Psi_{1}$ with $\Psi_{1} \in C^{\mu_{1}}\left(\mathcal{D}_{\text {tg }}^{\prime}\right)$. Plugging this equation in (4.10), implies that

$$
\Psi=x_{n}^{a+1} I_{a+1}\left(\Psi_{1}\right) \in x_{n}^{a+1} C^{\mu_{1}}\left(\mathcal{D}_{\mathrm{tg}}^{\prime}\right) .
$$

Summing up, we have proved that $\Phi=x_{n}^{-(a+1)} \Psi \in C^{\mu_{1}}\left(\mathcal{D}_{\mathrm{tg}}^{\prime}\right)$ and $x_{n} \partial_{x_{n}} \Phi=x_{n}^{-a} \partial_{x_{n}} \Psi-(a+1) \Phi \in C^{\mu_{1}}\left(\mathcal{D}_{\text {tg }}^{\prime}\right)$.

c) Inspecting the proof shows that the index $\mu$ and the Banach space $E$ such that (4.1) holds are independent of $\Psi$ and $f$. Moreover, each step in the preceding proof can be converted into an estimate ending with (4.2).

\section{Hölder estimates of $\Phi$.}

We continue the proof of Theorem 3.3, proving the Hölder smoothness of $\Phi$ and the estimate (3.19) of Theorem 3.3.

Proposition 5.1. Suppose that $\Psi$ and $f \in L^{p}([0, \delta] \times \omega), p>n$, are as in Theorem 3.3. Then for all relatively compact subset $\omega_{1} \subset \omega$ and all $\mu \leq 1-n / p, \Phi=x_{n}^{-(a+1)} \Psi \in C^{\mu}\left(\bar{\omega}_{1} \times[0, \delta]\right)$ and $x_{n} \Phi \in C^{\mu+1}\left(\bar{\omega}_{1} \times[0, \delta]\right)$, with

$$
\|\Phi\|_{C^{\mu}\left(\bar{\omega}_{1} \times[0, \delta]\right)}+\left\|x_{n} \Phi\right\|_{C^{\mu+1}\left(\bar{\omega}_{1} \times[0, \delta]\right)} \leq C\left(\|f\|_{L^{p}}+\|\Psi\|_{H^{1}}\right) .
$$

The equation (3.15) for $\Psi$ implies that $\Phi$ satisfies

$$
\mathcal{L} \Phi:=-x_{n} P_{2}\left(x, \partial_{x}\right) \Phi-Q_{1}\left(x, \partial_{x}\right) \Phi+R(x) \Phi=f,
$$

where

$$
\begin{aligned}
& P_{2}(x, \xi)=\sum_{j, k} p_{j, k}(x) \xi_{j} \xi_{k}, \\
& Q_{1}(x, \xi)=2(a+1) \sum_{j} p_{j, n}(x) \xi_{j}-a \sum_{j} p_{j}(x) \xi_{j}, \\
& R(x)=a(a+1)\left(p_{n}(x)-p_{n, n}(x)\right) / x_{n} .
\end{aligned}
$$

From (3.16), it follows that $R$ is smooth up to the boundary $\left\{x_{n}=0\right\}$ and that

$$
Q_{1}\left(x^{\prime}, 0, \xi\right)=(a+2) \sum_{j=1}^{n} p_{j, n}\left(x^{\prime}, 0\right) \xi_{j}
$$

Without loss of generality, we can also assume that $p_{n, n}=1$. 
Before proving the proposition above, we introduce several notations. For $\mu>0, \mu \notin \mathbb{N}$, we denote by $C^{\mu}\left(\overline{\mathbb{R}}_{+}^{n}\right)$ the space of bounded functions on $\mathbb{R}_{+}^{n}$ which are uniformly Hölder continuous with exponent $\mu$. Next, we denote by $C_{k}^{\mu}\left(\overline{\mathbb{R}}_{+}^{n}\right), k \in \mathbb{N}$, the space of functions $u \in C^{\mu}\left(\overline{\mathbb{R}}_{+}^{n}\right)$ such that $x_{n}^{j} u \in C^{\mu+j}$ for all $j \leq k$. $C_{\infty}^{\mu}$ is the intersection of the $C_{k}^{\mu}$. We refer to [BCM] for a definition of these spaces involving a Littlewood-Paley analysis and their extension to negative $\mu$ and real $k$. We also refer to the same paper for a precise definition of the spaces $C_{k}^{\mu, \nu}\left(\overline{\mathbb{R}}_{+}^{n}\right)$, where the second index $\nu \in \mathbb{R}$ measures the additional tangential smoothness. Finally, we introduce the spaces $C_{k}^{\mu,-\infty}=\bigcup_{\nu} C_{k}^{\mu, \nu}$.

Proposition 5.1 is a direct consequences of the results proved in [BCM], applied to second order equations (5.2). There are three assumptions in [BCM] to satisfy.

- (H1) The second order part $P_{2}$ is strongly elliptic.

- (H2) The indicial polynomial, $\lambda P_{2}\left(x^{\prime}, 0,0,1\right)+Q_{1}\left(x^{\prime}, 0,0,1\right)$ has no roots in the strip $\mu_{1} \leq \operatorname{Re} \lambda \leq \mu_{2}$.

- (H3) The differential operator $L^{0}=x_{n} P_{2}\left(x^{\prime}, 0, i \eta, \partial_{x_{n}}\right)+Q_{1}\left(x^{\prime}, 0, i \eta, \partial_{x_{n}}\right)$ is injective in the space $C_{\infty}^{\mu+1}\left(\overline{\mathbb{R}}_{+}\right)$for all $\eta \in \mathbb{R}^{n-1} \backslash\{0\}$ and all $x^{\prime}$.

1) For equation (5.2) the ellipticity assumption (H1) follows from (3.9).

2) By (5.3), the indicial polynomial is $(\lambda+a+2)$. An important feature is that its roots are independent of $x^{\prime}$. Since $a>0,(\mathrm{H} 2)$ is satisfied for all $-1<\mu_{1} \leq \mu_{2}$.

3) Using again (5.3), we have

$$
L^{0}=x_{n}\left(Z^{2}-\rho^{2}\right)+(a+2) Z=Z\left(x_{n} Z\right)+(a+1) Z-x_{n} \rho^{2},
$$

with

$$
\begin{aligned}
& Z=\partial_{x_{n}}+i \sum_{j<n} \eta_{j} p_{j, n}\left(0, x^{\prime}\right), \\
& \rho^{2}=\sum_{j, k<n} p_{j, k}\left(x^{\prime}, 0\right) \eta_{j} \eta_{k} .
\end{aligned}
$$

Since $\rho>0$ when $\eta \neq 0$, the bounded functions $u$ in the kernel of $L^{0}$ are smooth and rapidly decreasing at infinity. Moreover, they are smooth up 
to 0 , by the classical analysis of Fuchsian singularities. Thus, the following integration by parts are jusitified:

$$
0=\Re \int_{0}^{\infty} L^{0} u \bar{u} d x=-\int_{0}^{\infty} x_{n}\left(|Z u|^{2}+\rho^{2} u^{2}\right) d x_{n}-\frac{a+1}{2} \mid u\left(\left.0\right|^{2} .\right.
$$

This implies that $u=0$, hence that the spectral condition (H3) is satisfied if $\mu+1>0$.

Therefore we are in position to apply the results of [BCM].

Proof of Proposition 5.1. First, we note that, because $p>n$, the Sobolev embedding theorem implies that the right-hand side of (5.2) satisfies

$$
f \in L^{p} \subset C^{-n / p}(\omega \times[0, \delta]) .
$$

a) $\Phi$ vanishes for $x_{n} \geq \delta / 2$, so we can extend it by 0 for $x_{n} \geq \delta$. By proposition 4.1 we know that there are $\left.\left.\mu^{\prime} \in\right] 0,1 / 2\right]$ and a Banach space $E \subset \mathcal{D}^{\prime}(\omega)$ such that $\Phi \in C_{1}^{\mu^{\prime}}\left(\overline{\mathbb{R}}_{+} ; E\right)$. Therefore, Proposition 2.3.2 of BCM], implies that

$$
\chi \Phi \in C_{1}^{\mu^{\prime},-\infty}\left(\overline{\mathbb{R}}_{+}^{n}\right)
$$

if $\chi \in C^{\infty}(\omega)$ is equal to 1 on $\omega_{1}$. This implies that there is a (large negative) integer $\nu$ such that

$$
\chi \Phi \in C_{1}^{\mu_{1}+1, \nu}\left(\overline{\mathbb{R}}_{+}^{n}\right),
$$

if $\mu_{1}=\mu^{\prime}-1>-1$.

b) Let $\chi_{1} \in C_{0}^{\infty}(\omega)$, equal to 1 on $\omega_{1}$ and such that $\chi=1$ on a neighborhood of the support of $\chi_{1}$. There holds

$$
\mathcal{L} \chi_{1} \Phi=\chi_{1} f+\left[\mathcal{L}, \chi_{1}\right] \chi \Phi
$$

By (5.6), $\chi_{1} f \in C^{\mu-1}\left(\overline{\mathbb{R}}_{+}^{n}\right) \subset C^{\mu-1,0}\left(\overline{\mathbb{R}}_{+}^{n}\right)$ with $\mu=1-n / p$. By (5.7), $\left[\mathcal{L}, \chi_{1}\right] \chi \Phi \in C^{\mu_{1}+1, \nu}\left(\overline{\mathbb{R}}_{+}^{n}\right)$. Thus the right hand side of $(5.8)$ belongs to $C^{\mu_{2}, \nu}$ with $\mu_{2}=\mu-1<0<\mu_{1}+1=\mu^{\prime}$. Since the assumption (H2) is satisfied for the pair $\left(\mu_{1}, \mu_{2}\right)$, Theorem 4.1 in [BCM] implies that $\chi_{1} \Phi \in C_{1}^{\mu_{2}+1,-\infty}$, that is

$$
\chi_{1} \Phi \in C_{1}^{\mu_{2}+1, \nu_{1}}\left(\overline{\mathbb{R}}_{+}^{n}\right),
$$

for some $\nu_{1}$. 
c) Let $\chi_{2} \in C_{0}^{\infty}(\omega)$, equal to 1 on $\omega_{1}$ and such that $\chi=1$ on a neighborhood of the support of $\chi_{2}$. There holds

$$
\mathcal{L} \chi_{2} \Phi=\chi_{2} f+\left[\mathcal{L}, \chi_{2}\right] \chi_{1} \Phi .
$$

By $(5.6), \chi_{1} f \in C^{\mu-1}\left(\overline{\mathbb{R}}_{+}^{n}\right) \subset C^{\mu_{2}, 0}\left(\overline{\mathbb{R}}_{+}^{n}\right)$. By $(5.7),\left[\mathcal{L}, \chi_{1}\right] \chi \Phi \in C^{\mu_{2}+1, \nu_{1}}\left(\overline{\mathbb{R}}_{+}^{n}\right) \subset$ $C^{\mu_{2}, \nu_{1}+1}\left(\overline{\mathbb{R}}_{+}^{n}\right)$. By Theorem 5.2 in [BCM], we obtain that $\chi_{2} \Phi \in C_{1}^{\mu_{2}+1, \nu_{2}}$, with $\nu_{2}=\min \left(0, \nu_{1}+1\right)$.

Therefore, after finitely many iterations, we conclude that there is $\chi_{*} \in$ $C_{0}^{\infty}(\omega)$, equal to 1 on $\omega_{1}$ such that

$$
\chi_{*} \Phi \in C_{1}^{\mu_{2}+1,0}\left(\overline{\mathbb{R}}_{+}^{n}\right) .
$$

d) Finally, introducing $\chi_{b} \in C_{0}^{\infty}(\omega)$, equal to 1 on $\omega_{1}$ and such that $\chi_{*}=1$ on a neighborhood of the support of $\chi_{b}$ and writing the equation for $\chi_{b} \Phi$, Theorem 4.2 in BCM implies that $\chi_{b} \Phi \in C_{1}^{\mu_{2}+1}\left(\overline{\mathbb{R}}_{+}^{n}\right)=C_{1}^{\mu}\left(\overline{\mathbb{R}}_{+}^{n}\right)$ implying the proposition.

\section{$6 \quad L^{p}$ estimates}

In this section, we finish the proof of Theorem 3.3, proving the $L^{p}$ estimates (3.20). We recall that regularity result is performed in the general setting $\Omega \subset \mathbb{R}^{n}$ with $n \geq 1$. Given $\omega_{1}$ as in the theorem, consider $\omega_{2}$ relatively compact in $\omega$ such that $\bar{\omega}_{1} \subset \omega_{2}$. By Proposition 5.1, $\Phi$ is of class $C^{\mu}$ on $\omega_{2} \times[0, \delta]$ for $\mu \leq 1-n / p$. Consider $\chi \in C_{0}^{\infty}\left(\omega_{2}\right)$ with $\chi=1$ on $\omega_{1}$. Let $\phi=\chi \Phi \in C_{1}^{\mu}\left(\overline{\mathbb{R}}_{+}^{n}\right)$. Then

$$
\mathcal{L} \phi=g:=\chi f+[\mathcal{L}, \chi] \Phi \in L^{p}\left(\mathbb{R}_{+}^{n}\right) .
$$

Moreover, by Proposition 5.1, choosing $p_{0}>n$ and $\mu_{0}=1-n / p_{0}$, there holds for $f \in L^{p}, p \geq p_{0}$ :

$$
\begin{aligned}
\|\phi\|_{C_{1}^{\mu_{0}}\left(\overline{\mathbb{R}}_{+}^{n}\right)} & \leq C\left(\|f\|_{L^{p_{0}}}+\|\Psi\|_{H^{1}}\right), \\
\|g\|_{L^{p}\left(\mathbb{R}_{+}^{n}\right)} & \leq C\left(\|f\|_{L^{p}}+\|\Phi\|_{C_{1}^{\mu_{0}}\left(\overline{\mathbb{R}}_{+}^{n}\right)}\right) .
\end{aligned}
$$

Therefore, the $L^{p}$ estimates (3.20) follow from the next result.

Theorem 6.1. Let $\mu \in] 0,1\left[\right.$. Suppose that $\phi \in C_{1}^{\mu}\left(\overline{\mathbb{R}}_{+}^{n}\right)$ has compact support in $\omega \times\left[0, \delta\left[\right.\right.$ and $\mathcal{L} \phi=g \in L^{p}\left(\mathbb{R}_{+}^{n}\right)$. Then $\phi \in W_{1, \text { loc }}^{1, p}\left(\overline{\mathbb{R}}_{+}^{n}\right)$.

Moreover, there is a constant $C$ such that for all such $\phi$ and $p \in[2, \infty[$

$$
\left\|x_{n} \partial_{j} \partial_{k} \phi\right\|_{L^{p}\left(\mathbb{R}_{+}^{n}\right)}+\left\|\partial_{j} \phi\right\|_{L^{p}\left(\mathbb{R}_{+}^{n}\right)} \leq C\left(p\|g\|_{L^{p}\left(\mathbb{R}_{+}^{n}\right)}+\|\phi\|_{C_{1}^{\mu}}\right) .
$$




\subsection{Preliminary results}

In this section we recall some known results about Calderon-Zygmund operators ([St]). We consider operators $T$ acting in $L_{c o m p}^{\infty}\left(\overline{\mathbb{R}}_{+}^{n}\right)$ (the space of bounded functions with compact support) with kernel $T(x, y)$ locally integrable away from the diagonal $\{x=y\}$.

Proposition 6.2. Suppose that the kernel $K(x, y)$ satisfies on $\mathbb{R}_{+}^{n} \times \mathbb{R}_{+}^{n}$ :

$$
|K(x, y)| \leq \frac{C}{|x-y|^{n-1}}, \quad\left|\partial_{x} K(x, y)\right| \leq \frac{C}{|x-y|^{n}} .
$$

Then, the operator

$$
T f(x)=\int K(x, y) f(y) d y
$$

acts from $L_{\text {comp }}^{p}\left(\overline{\mathbb{R}}_{+}^{n}\right)$ to $C^{\mu}\left(\overline{\mathbb{R}}_{+}^{n}\right)$ for all $\mu<1-n / p$.

Proof. The first estimate for $K$ implies that $T$ maps $L_{\text {comp }}^{\infty}\left(\overline{\mathbb{R}}_{+}^{n}\right)$ to $L^{\infty}$. Moreover, the second estimate implies that

$$
\left|K(x, y)-K\left(x^{\prime}, y\right)\right| \leq C \frac{\left|x-x^{\prime}\right|}{|x-y|^{n}} \quad \text { for } \quad\left|x-x^{\prime}\right| \leq \frac{1}{2}|x-y| .
$$

Thus, interpolating with the first estimate yields

$$
\left|K(x, y)-K\left(x^{\prime}, y\right)\right| \leq C \frac{\left|x-x^{\prime}\right|^{\mu}}{|x-y|^{n-1+\mu}} \quad \text { for } \quad\left|x-x^{\prime}\right| \leq \frac{1}{2}|x-y| .
$$

We write

$$
\begin{aligned}
T f(x)-T f\left(x^{\prime}\right)= & \int_{|x-y| \geq 2\left|x-x^{\prime}\right|}\left(K(x, y)-K\left(x^{\prime}, y\right)\right) f(y) d y \\
& +\int_{|x-y| \leq 2\left|x-x^{\prime}\right|}\left(K(x, y)-K\left(x^{\prime}, y\right)\right) f(y) d y .
\end{aligned}
$$

By (6.6) the first integral is $O\left(\left|x-x^{\prime}\right|^{\mu}\right)$. Note that for $|x-y| \leq 2\left|x-x^{\prime}\right|$, there also holds $\left|x^{\prime}-y\right| \leq 3\left|x-x^{\prime}\right|$. Therefore, the second integral is

$$
O\left(\int_{|y| \leq 3\left|x-x^{\prime}\right|} \frac{d y}{|y|^{n-1}}\right)=O\left(\left|x-x^{\prime}\right|\right) .
$$


Proposition 6.3. Suppose that $T$ is a bounded operator in $L^{2}\left(\mathbb{R}_{+}^{n}\right)$ with kernel $K(x, y)$ satisfying for $x \neq y$ :

$$
|K(x, y)| \leq \frac{C}{|x-y|^{n}}, \quad\left|\partial_{x} K(x, y)\right| \leq \frac{C}{|x-y|^{n+1}} .
$$

Then $T$ maps $L^{p}\left(\mathbb{R}_{+}^{n}\right)$ to $L^{p}\left(\mathbb{R}_{+}^{n}\right)$ with norm $O(p)$ for all $p \in[2,+\infty[$.

Proof. The adjoint operator $T^{*}$ is bounded in $L^{2}$ and its kernel $K^{*}(x, y)=$ $\bar{K}(y, x)$ which therefore satisfies

$$
\left|K^{*}(x, y)\right| \leq \frac{C}{|x-y|^{n}}, \quad\left|\partial_{y} K^{*}(x, y)\right| \leq \frac{C}{|x-y|^{n+1}} .
$$

The Calderon-Zygmund theory implies that $T^{*}$ is bounded from $L^{1}$ to the space weak- $L^{1}$ (see e.g. [St]). Therefore, by Marcinkievic's interpolation theorem, $T^{*}$ maps $L^{p}$ to $L^{p}$ for $\left.\left.p \in\right] 1,2\right]$ with norm $O(1 /(p-1)$ (same reference). By duality, this implies that $T$ maps $L^{p}$ to $L^{p}$ for $p \in[2, \infty[$ with norm $O(p)$.

\subsection{Parametrices}

Recall that $\mathcal{L} \approx-x_{n} P_{2}-Q_{1}$, see (5.2). For $y \in \bar{\omega}$, we denote by $\mathcal{L}_{y}$ the operator

$$
\mathcal{L}_{y}\left(x, \partial_{x}\right):=-x_{n} P_{2}\left(y, \partial_{x}\right)-(a+2)\left(\partial_{x_{n}}+\sum_{j<n} p_{j, n}(y) \partial_{j}\right)
$$

As above, we have assumed as we may that $p_{n, n}=1$. For a given $y$, there is a linear transformation

$$
\tilde{x}=T(y) x, \text { with } \tilde{x}_{n}=x_{n}
$$

such that, in these variables $\mathcal{L}_{y}$ is transformed to

$$
\widetilde{\mathcal{L}}=-\tilde{x}_{n} \Delta_{\tilde{x}}-(a+2) \partial_{\tilde{x}_{n}} .
$$

According to [GS, Lemme 1, the fundamental solution of $\widetilde{\mathcal{L}}$ is

$$
\widetilde{E}(\tilde{x}, \tilde{y})=\int_{0}^{1} \tilde{F}(\tilde{x}, \tilde{y}, \theta) d \theta,
$$

with

$$
\begin{aligned}
\tilde{F}(\tilde{x}, \tilde{y}, \theta) & =\gamma\left(\tilde{y}_{n}\right)^{a+1} A^{-(a+n)}(\theta(1-\theta))^{a / 2} \\
A^{2}(\tilde{x}, \tilde{y}, \theta) & =\theta D^{2}+(1-\theta) \check{D}^{2}
\end{aligned}
$$


with $\gamma$ some constant depending on $a$ and

$$
D^{2}=\left|\tilde{x}_{n}-\tilde{y}_{n}\right|^{2}+\left|\tilde{x}^{\prime}-\tilde{y}^{\prime}\right|^{2}, \quad \check{D}^{2}=\left|\tilde{x}_{n}+\tilde{y}_{n}\right|^{2}+\left|\tilde{x}^{\prime}-\tilde{y}^{\prime}\right|^{2} .
$$

More precisely, for $\varepsilon \in] 0,1[$, let

$$
\widetilde{E}^{\varepsilon}(\tilde{x}, \tilde{y})=\int_{0}^{1-\varepsilon} \tilde{F}(\tilde{x}, \tilde{y}, \theta) d \theta .
$$

This function is singular only on $\tilde{y}_{n}=0$ and there holds

$$
\widetilde{L}\left(\tilde{x}, \partial_{\tilde{x}}\right) \tilde{E}^{\varepsilon}(\tilde{x}, \tilde{y})=\widetilde{G}^{\varepsilon}(\tilde{x}, \tilde{y})
$$

where

$$
\tilde{G}^{\varepsilon}(\tilde{x}, \tilde{y})=2(n-2+a) \gamma \tilde{y}_{n}^{a+2} A^{-(a+2+n)}(\varepsilon(1-\varepsilon))^{(a+2) / 2} .
$$

According to GS, Appendix A, there holds :

Lemma 6.4. $\tilde{G}^{\varepsilon}(\tilde{x}, \tilde{y})$ is an approximation of the identity as $\varepsilon$ tends to zero: it is nonnegative, converges uniformly to 0 on compacts of $\overline{\mathbb{R}}_{+}^{d} \times \overline{\mathbb{R}}_{+}^{d} \backslash\{\tilde{x}=\tilde{y}\}$ and for all bounded open set $\Omega \subset \mathbb{R}_{+}^{n}$ and all $\tilde{x} \in \Omega$

$$
\lim _{\varepsilon \rightarrow 0} \int_{\Omega} \tilde{G}^{\varepsilon}(\tilde{x}, \tilde{y}) d \tilde{y}=1 .
$$

From $\widetilde{E}$, we derive fundamental solutions for the operators $\mathcal{L}_{z}$ :

$$
E_{z}(x, y)=\left|\operatorname{det} T^{\prime}(z)\right| E(T(z) x, T(z) y)
$$

and their approximate versions

$$
E_{z}^{\varepsilon}(x, y)=\left|\operatorname{det} T^{\prime}(z)\right| E^{\varepsilon}(T(z) x, T(z) y)
$$

Finally, we define the parametrices

$$
E(x, y)=E_{y}(x, y), \quad E^{\varepsilon}(x, y)=E_{y}^{\varepsilon}(x, y) .
$$

Similarly, we define the $G_{z}^{\varepsilon}(x, y)$ and $G^{\varepsilon}(x, y)$.

Proposition 6.5. Suppose that $a \geq 1$. For $k \in \mathbb{N}$, there is a constant $C_{k}$ such that for $\left.(x, y) \in \mathbb{R}_{+}^{n} \times \omega, x \neq y, \varepsilon \in\right] 0,1[$, there holds

$$
\left|\nabla_{x}^{k} E^{\varepsilon}(x, y)\right| \leq \frac{C_{k}}{|x-y|^{n+k-1}},
$$

and for $k \geq 1$

$$
\left|x_{n} \nabla_{x}^{k} E^{\varepsilon}(x, y)\right| \leq \frac{C_{k}}{|x-y|^{n+k-2}} .
$$

The same estimates hold for $E$. 
Proof. It is sufficient to prove the estimates for $E_{z}^{\varepsilon}(x, y)$ and therefore for $\widetilde{E}(\tilde{x}, \tilde{y})$. For simplicity, we drop the tildes in the proof below. On $\mathbb{R}_{+}^{n} \times \mathbb{R}_{+}^{n}$, there holds

$$
A \geq|x-y|, \quad A \geq(1-\theta)^{1 / 2}\left(x_{n}+y_{n}\right) .
$$

Hence, for $a \geq-1$,

$$
y_{n}^{a+1} A^{-(a+n)} \leq|x-y|^{1-n}(1-\theta)^{-(a+1) / 2},
$$

implying

$$
|F(x, y, \theta)| \leq \gamma|x-y|^{1-n} \theta^{a / 2}(1-\theta)^{-1 / 2} .
$$

Thus, integrating in $\theta$,

$$
\left|E^{\varepsilon}(x, y)\right| \leq C|x-y|^{1-n}
$$

Estimates on the derivatives of $E$. Differentiating $k$ times $A^{2}$, one proves by induction that

$$
\left|\nabla_{x}^{k} A\right| \leq C_{k} A^{1-k}
$$

Thus

$$
\left|\nabla_{x}^{k} F\right| \leq C_{k} y_{n}{ }^{a+1} A^{-(a+k+n)}(\theta(1-\theta))^{a / 2} .
$$

Using (6.22) yields

$$
\left|\nabla_{x}^{k} F(x, y)\right| \leq C|x-y|^{1-k-n} \theta^{a / 2}(1-\theta)^{-1 / 2},
$$

implying

$$
\left|\nabla_{x}^{k} E^{\varepsilon}(x, y)\right| \leq C|x-y|^{1-k-n} .
$$

Sharper estimates of $x_{n} \nabla_{x}^{k} E$. There holds

$$
A^{2}=|x-y|^{2}+4(1-\theta) x_{n} y_{n} .
$$

Let us assume first that

$$
x_{n} y_{n} \leq 2|x-y|^{2} .
$$

Then,

$$
\left(x_{n}+y_{n}\right)^{2} \leq\left(x_{n}-y_{n}\right)^{2}+4 x_{n} y_{n} \leq C|x-y|^{2} .
$$


In this case, we use that $A \geq|x-y|$, without loosing any information and

$$
\left|x_{n} \nabla_{x}^{k} F(x, y)\right| \leq C x_{n} y_{n}^{a+1}|x-y|^{-(n+k+a)}(\theta(1-\theta))^{a / 2}
$$

Integrating in $\theta$ yields

$$
\left|x_{n} \nabla_{x}^{k} E(x, y)\right| \leq C x_{n} y_{n}^{a+1}|x-y|^{-(n+k+a)} .
$$

With (6.28), $x_{n} y_{n}^{a+1} \leq C|x-y|^{a+2}$, therefore

$$
\left|x_{n} \nabla_{x}^{k} E^{\varepsilon}(x, y)\right| \leq C|x-y|^{-(n+k-2)} .
$$

Consider next the case

$$
x_{n} y_{n} \geq 2|x-y|^{2} .
$$

In this case,

$$
5 x_{n} y_{n} \geq 2 x_{n}^{2}+2 y_{n}^{2} \quad \Rightarrow \quad \frac{1}{C} \leq \frac{x_{n}}{y_{n}} \leq C .
$$

Let

$$
\left.\left.\rho:=\frac{|x-y|^{2}}{4 x_{n} y_{n}} \in\right] 0, \frac{1}{2}\right] .
$$

We use the estimates

$$
\begin{array}{ll}
A^{2} \geq|x-y|^{2} & \text { when } \quad 1-\theta \leq \rho, \\
A^{2} \geq 4(1-\theta) x_{n} y_{n} & \text { when } \quad 1-\theta \geq \rho .
\end{array}
$$

Therefore,

$$
\begin{aligned}
\left|x_{n} \nabla_{x}^{k} E^{\varepsilon}(x, y)\right| \leq C & \frac{x_{n} y_{n}^{a+1}}{|x-y|^{n+k+a}} \int_{1-\rho}^{1}(\theta(1-\theta))^{a / 2} d \theta \\
& +C \frac{x_{n} y_{n}^{a+1}}{\left(x_{n} y_{n}\right)^{(n+k+a) / 2}} \int_{0}^{1-\rho} \theta^{a / 2}(1-\theta)^{-(n+k) / 2} d \theta
\end{aligned}
$$

Thus

$$
\begin{aligned}
\left|x_{n} \nabla_{x}^{k} E^{\varepsilon}(x, y)\right| \leq C & \frac{x_{n} y_{n}^{a+1}}{|x-y|^{n+k+a}} \rho^{a / 2} \\
& +C \frac{x_{n} y_{n}^{a+1}}{\left(x_{n} y_{n}\right)^{(n+k+a) / 2}} \rho^{1-(n+k) / 2} .
\end{aligned}
$$


For the last estimate we have used that $n+k>2$. Therefore,

$$
\begin{aligned}
&\left|x_{n} \nabla_{x}^{k} E^{\varepsilon}(x, y)\right| \leq C \frac{x_{n} y_{n}^{a+1}}{|x-y|^{n+k+a}} \frac{|x-y|^{a+2}}{\left(x_{n} y_{n}\right)^{(a+2) / 2}} \\
&+C \frac{x_{n} y_{n}^{a+1}}{\left(x_{n} y_{n}\right)^{(n+k+a) / 2}} \frac{\left(x_{n} y_{n}\right)^{(n+k-2) / 2}}{|x-y|^{(n+k-2)}} \\
&\left|x_{n} \nabla_{x}^{k} E^{\varepsilon}(x, y)\right| \leq C \frac{y_{n}^{a / 2}}{x_{n}^{a / 2}} \frac{1}{|x-y|^{n+k-2}} .
\end{aligned}
$$

Together with (6.31) this implies

$$
\left|x_{n} \nabla_{x}^{k} E^{\varepsilon}(x, y)\right| \leq C \frac{1}{|x-y|^{n+k-2}} .
$$

This finishes the proof of the proposition.

Lemma 6.6. The following identity holds

$$
\mathcal{L}\left(x, \partial_{x}\right) E^{\varepsilon}(x, y)=G^{\varepsilon}(x, y)+K^{\varepsilon}(x, y)
$$

where the kernels $K^{\varepsilon}$ satisfy uniformly in $\left.\varepsilon \in\right] 0,1[$ :

$$
\left|K^{\varepsilon}(x, y)\right| \leq \frac{C}{|x-y|^{n-1}}, \quad\left|\partial_{x} K^{\varepsilon}(x, y)\right| \leq \frac{C}{|x-y|^{n}} .
$$

Moreover, the kernels $K^{\varepsilon}$ converge uniformly on compacts of $\{x \neq y\}$ to $K(x, y)$ which satisfy (6.35) outside the diagonal.

Proof. The definition of $E^{\varepsilon}$ implies that it is singular only on $y_{n}=0$. Moreover,

$$
\mathcal{L}\left(x, \partial_{x}\right) E^{\varepsilon}(x, y)=\mathcal{L}_{y}\left(x, \partial_{x}\right) E^{\varepsilon}(x, y)+K^{\varepsilon}(x, y)=G^{\varepsilon}(x, y)+K^{\varepsilon}(x, y)
$$

where

$$
\begin{aligned}
K^{\varepsilon}(x, y)=x_{n} \sum & \left(p_{j, k}(y)-p_{j, k}(x)\right) \partial_{x_{j}} \partial_{x_{k}} E^{\varepsilon} \\
& +\sum\left((a+2) p_{j, n}(y)-q_{j}(x)\right) \partial_{x_{j}} E^{\varepsilon}+R(x) E^{\varepsilon} .
\end{aligned}
$$

where the $q_{j}$ are the coefficients of $Q_{1}$. In the first sum, the coefficient of $x_{n} \partial_{x_{j}} \partial_{x_{k}} E^{\varepsilon}$ is $O(|x-y|)$. By (5.3), the coefficient of $\partial_{x_{j}} E^{\varepsilon}$ in the second sum is $O\left(x_{n}\right)+O(|x-y|)$. Together with Proposition 6.5, this implies the estimates (6.35).

From (6.36), it is clear that $K^{\varepsilon}$ converges to a kernel $K$ which satisfies (6.35). 
Lemma 6.7. The kernels $G^{\varepsilon}(x, y)$ are non negative, converge to 0 as $\varepsilon$ tends to 0 uniformly on compacts subsets of $\left(\overline{\mathbb{R}}_{+}^{n} \times \bar{\omega}\right) \backslash\{x=y\}$. Moreover the integrals

$$
\int_{\omega \times] 0, \delta[} G^{\varepsilon}(x, y) d y
$$

are uniformly bounded for $\varepsilon \in] 0,1[$ and $x$ in a compact subset of $\omega \times] 0, \delta[$, and converge to 1 as $\varepsilon$ tends to 0 .

Proof. By (6.16), (6.26) and (6.10), it follows that

$$
\begin{aligned}
G_{z}^{\varepsilon}(x, y) & \left.=\left|\operatorname{det} T^{\prime}(z)\right| \widetilde{G}^{\varepsilon}(T(z) x, T(z) y)\right) \\
& =\left|\operatorname{det} T^{\prime}(z)\right| \frac{y_{n}^{a+2}(\varepsilon(1-\varepsilon))^{(a+2) / 2}}{\left(|T(z)(x-y)|^{2}+4 \varepsilon x_{n} y_{n}\right)^{(a+n+2) / 2}}
\end{aligned}
$$

is nonnegative. Thus

$$
G_{z}^{\varepsilon}(x, y)+\left|\partial_{z} G_{z}^{\varepsilon}(x, y)\right| \leq \frac{C y_{n}^{a+2} \varepsilon^{(a+2) / 2}}{\left(|x-y|^{2}+\varepsilon x_{n} y_{n}\right)^{(a+n+2) 2}} .
$$

In particular, $G^{\varepsilon}(x, y)=G_{y}^{\varepsilon}(x, y)$ is also dominated by the same bound. implying that $G^{\varepsilon}(x, y) \rightarrow 0$ as $\varepsilon \rightarrow 0$ when $x \neq y$, uniformly on compact subsets.

Integrating in the tangential variables first implies that

$$
\int_{\omega \times] 0, \delta[} G^{\varepsilon}(x, y) d y \leq \int_{0}^{L} \frac{C y_{n}^{a+2} \varepsilon^{(a+2) / 2}}{\left(\left|x_{n}-y_{n}\right|^{2}+\varepsilon x_{n} y_{n}\right)^{(a+3) / 2}} d y_{n} .
$$

The integral over $\left\{\left|x_{n}-y_{n}\right| \geq x_{n} / 2\right.$ tends to zero, uniformly for $x_{n}$ in a compact subset of $] 0, \delta]$. The integral over the remaining interval $\left\{\left|x_{n}-y_{n}\right| \leq\right.$ $x_{n} / 2$ is bounded by

$$
\begin{aligned}
\int_{\frac{x_{n}}{2}}^{\frac{3 x_{n}}{2}} \frac{C x_{n}^{a+2} \varepsilon^{(a+2) / 2}}{\left(\left|x_{n}-y_{n}\right|^{2}+\varepsilon x_{n}^{2}\right)^{(a+3) 2}} d y_{n} & =\int_{-\frac{1}{2}}^{\frac{1}{2}} \frac{C \varepsilon^{(a+2) / 2}}{\left(\left|y_{n}\right|^{2}+\varepsilon\right)^{(a+3) 2}} d y_{n} \\
& \leq \int_{\mathbb{R}} \frac{C}{\left(\left|y_{n}\right|^{2}+1\right)^{(a+3) 2}} d y_{n}<+\infty .
\end{aligned}
$$

Moreover, by (6.37) there holds

$$
\left|G_{x}^{\varepsilon}(x, y)-G_{y}^{\varepsilon}(x, y)\right| \leq \frac{C|x-y| y_{n}^{a+2} \varepsilon^{(a+2) / 2}}{\left(|x-y|^{2}+\varepsilon x_{n} y_{n}\right)^{(a+n+2) 2}} .
$$


This implies that

$$
\lim _{\varepsilon \rightarrow 0} \int_{\omega \times] 0, \delta[}\left|G_{x}^{\varepsilon}(x, y)-G_{y}^{\varepsilon}(x, y)\right| d y=0 .
$$

By Lemma 6.4 (see GS]), for $x \in \Omega$ there holds

$$
\lim _{\varepsilon \rightarrow 0} \int_{\omega \times] 0, \delta[} G_{x}^{\varepsilon}(x, y) d y=1,
$$

implying that

$$
\lim _{\varepsilon \rightarrow 0} \int_{\omega \times] 0, \delta[} G^{\varepsilon}(x, y) d y=1 .
$$

This finishes the proof of the lemma.

We denote by $\mathbf{E}, \mathbf{E}^{\varepsilon}$ etc the operator with kernel $E, E^{\varepsilon}$ etc. In particular, since the kernels are smooth for $y_{n}>0$ and bounded on $\mathbb{R}_{+}^{n} \times \mathbb{R}_{+}^{n}$, the operators $\mathbf{E}^{\varepsilon}, \mathbf{G}^{\varepsilon}$ and $\mathbf{K}^{\varepsilon}$ are defined from the space of integrable functions with support in $\omega \times[0, \delta]$ to the space of $C^{\infty}\left(\overline{\mathbb{R}}_{+}^{n}\right)$. The lemma above implies that

$$
\mathcal{L} \mathbf{E}^{\varepsilon}=\mathbf{G}^{\varepsilon}+\mathbf{K}^{\varepsilon} .
$$

Moreover, the estimates $(6.20)$ and $(6.35)$ imply that the operators $\mathbf{E}^{\varepsilon}$ [resp. $\left.\mathbf{K}^{\varepsilon}\right]$ are uniformly bounded from $L^{p}(\omega \times] 0, \delta[)$ to $L_{l o c}^{p}\left(\overline{\mathbb{R}}_{+}^{n}\right)$ and converge in the strong topology to the operators $\mathbf{E}$ [resp. $\mathbf{K}$ ] defined by the kernels $E$ [resp. $K]$.

Proposition 6.8. The operator $\mathbf{E}$ maps $L^{p}(\omega \times] 0, \delta[)$ to $W_{1, l o c}^{1, p}\left(\overline{\mathbb{R}}_{+}^{n}\right)$. For all relatively compact open set $\omega_{1} \subset \omega$, all $\delta^{\prime}<\delta$ and for all $\chi \in C_{0}^{\infty}(\omega \times[0, \delta[)$ such that $\chi=1$ on a neighborhood of $\bar{\omega}_{1} \times[0, \delta]$, there is a constant $C$ such that for all $p \in\left[2,+\infty\left[\right.\right.$ and all $g \in L^{p}$ supported in $\left.\omega_{1} \times\right] 0, \delta^{\prime}[$, there holds

$$
\left\|x_{n} \chi \partial_{j} \partial_{k} \mathbf{E} g\right\|_{L^{p}\left(\mathbb{R}_{+}^{n}\right)}+\left\|\chi \partial_{j} \mathbf{E} g\right\|_{L^{p}\left(\mathbb{R}_{+}^{n}\right)} \leq p C\|\mathbf{E} g\|_{L^{p}\left(\mathbb{R}_{+}^{n}\right)} .
$$

Moreover, for all $p>n$ and $g \in L^{p}$ supported in $\omega_{1} \times\left[0, \delta^{\prime}\right]$ and all $\mu<1-n / p$, there holds

$$
\mathcal{L} \mathbf{E} g-g=\mathbf{K} g \in C_{1, l o c}^{\mu}\left(\overline{\mathbb{R}}_{+}^{n}\right) .
$$

Proof. By Propositions 6.5, for $g \in L^{p}(\omega \times] 0, \delta[), \mathbf{E}^{\varepsilon} g$ and $\mathbf{K}^{\varepsilon} g$ are uniformly bounded in $L^{p}\left(\overline{\mathbb{R}}_{+}^{n}\right)$ and converge in $L_{l o c}^{p}$ to $\mathbf{E} g$ and $\mathbf{K} g$ respectively. 
Suppose that $g$ is continuous with compact support in $\omega \times] 0, \delta[$. Then, by Lemma 6.7, $\mathbf{G}^{\varepsilon} g$ converges to $g$ as $\varepsilon$ tends to 0 . This shows that the identity (6.41) is satisfied, in the sense of distributions, for $g \in C^{0}$ with compact support in $\omega \times] 0, \delta\left[\right.$. By density, this identity extends to $g \in L^{2}(\omega \times] 0, \delta[)$. In particular, $\mathbf{E} g \in L_{l o c}^{2}\left(\overline{\mathbb{R}}_{+}^{n}\right)$ and $\mathcal{L} \mathbf{E} g \in L_{l o c}^{2}\left(\overline{\mathbb{R}}_{+}^{n}\right)$. Using the regularity properties of $L$ proved in $\left[\overline{\mathrm{BQ}}\right.$, this implies that $\mathbf{E}$ maps $L^{2}(\omega \times] 0, \delta[)$ into the space $H_{1, l o c}^{1}\left(\omega \times\left[0, \delta[)\right.\right.$ of functions $u \in H_{l o c}^{1}\left(\omega \times\left[0, \delta[)\right.\right.$ such that $x_{n} u \in$ $H_{l o c}^{2}(\omega \times[0, \delta[)$.

In particular, this proves that for $\chi_{1}$ and $\chi_{2}$ in $C_{0}^{\infty}\left(\overline{\mathbb{R}}_{+}^{n}\right)$, the operators $\chi_{1} x_{n} \partial_{j} \partial_{k} \mathbf{E} \chi_{2}$ and $\chi_{1} \partial_{j} \mathbf{E} \chi_{2}$ are bounded from $L^{2}\left(\mathbb{R}_{+}^{n}\right)$ to $L^{2}\left(\mathbb{R}_{+}^{n}\right)$. Together with the estimates of Proposition 6.5 and with Proposition 6.3, this implies that $\mathbf{E}$ satisfies the properties listed in the proposition and the estimates (6.40).

The identity (6.41) holds for $g \in L^{p}$ thus for $g \in L^{\infty}$. The properties of $\mathbf{K}$ follow from Proposition 6.2 together with the estimates of Lemma 6.6.

\subsection{Proof of Theorem 6.1}

By assumption, $\phi$ and $g=\mathcal{L} \phi$ have compact support in $\omega \times[0, \delta[$. Let $\chi$ and $\tilde{\chi}$ in $C_{0}^{\infty}(\omega \times[0, \delta[)$ such that $\chi=1$ on a neighborhood of the support of $\phi$ and $\tilde{\chi}=1$ on a neighborhood of the support of $\chi$.

By Propositions 6.5 and 6.2, $\tilde{\chi} \mathbf{E} g \in C_{1}^{\mu}\left(\overline{\mathbb{R}}_{+}^{n}\right)$. Let $\psi=\chi \mathbf{E} g$, which satisfies the estimates (6.40). Moreover,

$$
\mathcal{L} \psi=\chi \mathcal{L} \mathbf{E} g+[\mathcal{L}, \chi] \mathbf{E} g=g+\chi \mathbf{K} g+[\mathcal{L}, \chi] \tilde{\chi} \mathbf{E} g .
$$

In particular,

$$
\mathcal{L}(\phi-\psi)=\chi \mathbf{K} g+[\mathcal{L}, \chi] \tilde{\chi} \mathbf{E} g .
$$

By Proposition 6.8, $\chi \mathbf{K} g \in C_{1}^{\mu}\left(\overline{\mathbb{R}}_{+}^{n}\right)$ and since $\tilde{\chi} \mathbf{E} g \in C_{1}^{\mu}\left(\overline{\mathbb{R}}_{+}^{n}\right)$, the commutator $[\mathcal{L}, \chi] \tilde{\chi} \mathbf{E} g$ also belongs to $C^{\mu}\left(\overline{\mathbb{R}}_{+}^{n}\right)$. Thus the right hand side $h$ of (6.42) is in $C^{\mu}\left(\overline{\mathbb{R}}_{+}^{n}\right)$

We are now in position to apply the results of [GS] (or BCM]), which imply that

$$
\phi-\psi \in C_{l o c}^{\mu+1}\left(\overline{\mathbb{R}}_{+}^{n}\right), \quad x_{n}(\phi-\psi) \in C_{l o c}^{\mu+2}\left(\overline{\mathbb{R}}_{+}^{n}\right) .
$$

Therefore,

$$
\begin{aligned}
\left\|x_{n} \partial_{j} \partial_{k}(\phi-\psi)\right\|_{L^{\infty}\left(\mathbb{R}_{+}^{n}\right)}+ & \left\|\partial_{j}(\phi-\psi)\right\|_{L^{\infty}\left(\mathbb{R}_{+}^{n}\right)} \leq \\
& C\left(\|h\|_{C^{\mu}\left(\overline{\mathbb{R}}_{+}^{n}\right)}+\|(\phi-\psi)\|_{C_{1}^{\mu}\left(\overline{\mathbb{R}}_{+}^{n}\right)}\right)
\end{aligned}
$$


with $h$ the right-hand side of (6.42).

Together with the estimates of Proposition 6.8 for the $L^{p}$ norm of $x_{n} \partial_{j} \partial_{k} \psi$ and $\partial_{j} \psi$, this implies the theorem.

\section{Proof of global existence, uniqueness and regu- larity theorem.}

Regularity of weak solutions. Suppose that $(v, \omega)$ is a weak solution in the sense of Definition 2.1. Then $v \in L^{\infty}\left(0, T ; W^{1, p}(\Omega)\right)$ for all $p<$ $+\infty$ from the elliptic regularity theorem. Therefore, using Di-Perna and Lions renormalization technics for transport equation (1.3), implies that $\omega \in C\left(0, T ; L^{r}(\Omega)\right)$, hence that $v \in C\left(0, T ; W^{1, r}(\Omega)\right)$ for all $r<+\infty$. The other properties of $v$ also follow from Theorem 2.3.

Existence of a global weak solution. We construct a global weak solution as the inviscid limit of solutions to a Navier-Stokes system with artificial viscosity and boundary conditions.

Global existence for a viscous vorticity-stream function formulation. We define $b_{\varepsilon}=b+\varepsilon$ and consider the following system

$$
\left\{\begin{array}{l}
\partial_{t}\left(b^{\varepsilon} \omega^{\varepsilon}\right)+b v^{\varepsilon} \cdot \nabla \omega^{\varepsilon}-\varepsilon \operatorname{div}\left(b^{\varepsilon} \nabla \omega^{\varepsilon}\right)=0 \text { in } \Omega, \\
\left.b^{\varepsilon} \omega^{\varepsilon}\right|_{t=0}=b^{\varepsilon} \omega_{0} \text { in } \Omega,\left.\quad \omega^{\varepsilon}\right|_{\partial \Omega}=0, \\
-\operatorname{div}\left(\frac{1}{b} \nabla \Psi^{\varepsilon}\right)=b^{\varepsilon} \omega^{\varepsilon} \text { in } \Omega,\left.\quad \Psi^{\varepsilon}\right|_{\partial \Omega}=0, \\
v=\frac{1}{b} \nabla^{\perp} \Psi^{\varepsilon} .
\end{array}\right.
$$

The existence of a global weak solution $\left(v^{\varepsilon}, \omega^{\varepsilon}\right)$ for $\omega_{0} \in L^{2}(\Omega)$ follows standard techniques for equations of Navier-Stokes type since $b^{\varepsilon}$ is a strictly positive function, see [LOT]. From the a-priori estimates, we get the following uniform bounds with respect to $\varepsilon$

$$
\sqrt{b}^{\varepsilon} \omega^{\varepsilon} \in L^{\infty}\left(0, T ; L^{2}(\Omega)\right), \quad \sqrt{b}^{\varepsilon} \nabla \omega^{\varepsilon} \in L^{2}\left(0, T ; L^{2}(\Omega)\right) .
$$

Moreover $\sqrt{b} v^{\varepsilon} \in L^{\infty}\left(0, T ; L^{2}(\Omega)\right)$ uniformly.

The viscous limit $\varepsilon \rightarrow 0$. Let us now assume that $\omega_{0} \in L^{\infty}(\Omega)$. Since $\omega^{\varepsilon} \in L^{\infty}\left(0, T ; L^{2}(\Omega)\right) \cap L^{2}\left(0, T ; H_{0}^{1}(\Omega)\right)$, using the elliptic regularity for degenerate elliptic equation, we get $\partial_{t} \omega^{\varepsilon} \in L^{\infty}\left(0, T ; H^{-1}(\Omega)\right)$ and thus $\omega^{\varepsilon} \in$ 
$C\left([0, T) ; L^{2}(\Omega)\right)$. We multiply the viscous equation by $\left|T_{R}\left(\omega^{\varepsilon}\right)\right|^{p-2} T_{R}\left(\omega^{\varepsilon}\right)$ where $T_{R}\left(\omega^{\varepsilon}\right)=\max \left(\min \left(\omega^{\varepsilon}, R\right),-R\right)$ for $R>0$, and we get

$$
\begin{array}{r}
\frac{1}{p} \int_{\Omega} b^{\varepsilon}\left|T_{R}\left(\omega^{\varepsilon}\right)(t)\right|^{p}+\varepsilon(p-1) \int_{0}^{t} d s \int_{\Omega} b^{\varepsilon}\left|\nabla T_{R}\left(\omega^{\varepsilon}\right)\right|^{2}|\omega|^{p-2} d x \\
=\frac{1}{p} \int_{\Omega} b^{\varepsilon}\left|T_{R}\left(\omega_{0}\right)\right|^{p} d x .
\end{array}
$$

Therefore

$$
\left\|b^{1 / p} T_{R}(\omega)(t)\right\|_{L^{p}(\Omega)} \leq\left\|b^{1 / p} T_{R}\left(\omega_{0}\right)\right\|_{L^{p}(\Omega)} .
$$

Letting $R$ go to $+\infty$, this gives

$$
\left\|\left(b^{\varepsilon}\right)^{1 / p} \omega^{\varepsilon}\right\|_{L^{p}(\Omega)} \leq\left\|\left(b^{\varepsilon}\right)^{1 / p} \omega_{0}\right\|_{L^{p}(\Omega)} .
$$

and since $\omega_{0} \in L^{\infty}$ and the estimate is uniform

$$
\left\|\omega^{\varepsilon}\right\|_{L^{\infty}(\Omega)} \leq\left\|\omega_{0}\right\|_{L^{\infty}(\Omega)} .
$$

Using the estimate obtained in the main estimate part we also get

$$
\left\|v^{\varepsilon}\right\|_{L^{4}(\Omega)} \leq C\left(\left\|\omega^{\varepsilon}\right\|_{L^{\infty}(\Omega)}+\left\|\sqrt{b} v^{\varepsilon}\right\|_{L^{2}(\Omega)}\right) .
$$

Next we note that the set $\left.\left.\left\{b^{\varepsilon} \omega^{\varepsilon}, \varepsilon \in\right] 0,1\right]\right\}$ is relatively compact in $C^{0}\left([0, \infty) ; L_{w}^{2}(\Omega)\right)$ and in $C^{0}\left([0, \infty) ; L_{w *}^{\infty}(\Omega)\right)$ as in LOT. Thus $\left\{b^{\varepsilon} \omega^{\varepsilon}\right\}$ is a relatively compact set in $L_{\text {loc }}^{2}\left([0, \infty) ; H^{-1}(\Omega)\right)$. Therefore $\left\{\sqrt{b} v^{\varepsilon}\right\}$ is relatively compact in $L_{\text {loc }}^{2}\left([0, \infty) ; L^{2}(\Omega)\right)$. This allows to pass to the limit in the viscous formulation and to get the global existence of a weak solution of the inviscid stream-vorticity formulation.

\section{Uniqueness of weak solutions.}

Proof. Let $v_{1}$ and $v_{2}$ be two solutions of (1.1). Then $v=v_{1}-v_{2}$ satisfies

$$
\partial_{t} v+v_{2} \cdot \nabla v+\nabla p=-v \cdot \nabla v_{1}
$$

Therefore,

$$
\frac{d}{d t}\|\sqrt{b} v\|_{L^{2}}^{2} \leq 2 \int_{\Omega} b|v|^{2}\left|\nabla v_{1}\right|
$$


If $\nabla v_{1} \in L^{\infty}$, this clearly implies that $v=0$. But in general, this $L^{\infty}$ estimate is not available, but following [Y]], sharp $L^{p}$ estimates can be substituted. By Theorem 2.3,

$$
C:=\sup _{p \geq 3}\left\{\frac{1}{p}\left(\int_{\Omega}\left|\nabla u_{1}\right|^{p}\right)^{\frac{1}{p}}\right\}<\infty
$$

In the right hand side of (7.2), we use Young's inequality with

$$
\left|\nabla v_{1}\right| \in L^{p}, \quad b^{1 / p^{\prime}}|v|^{2 / p^{\prime}} \in L^{p^{\prime}}, \quad b^{1-1 / p^{\prime}}|v|^{2-2 / p^{\prime}}=|b v|^{2 / p} \in L^{\infty},
$$

implying that $y(t):=\|\sqrt{b} v(t)\|_{L^{2}}^{2}$ satisfies for all $p \geq 3$ :

$$
\partial_{t} y(t) \leq p C M^{2 / p}\{y(t)\}^{1 / p^{\prime}}
$$

with $M=\|\sqrt{b} v\|_{L^{\infty}}+\left\|\sqrt{b} v_{2}\right\|_{L^{\infty}}$. Optimizing in $p$ yields

$$
\partial_{t} y(t) \leq e C y(t) \frac{1}{\ln \left(M^{2} / y(t)\right)} .
$$

Since $y(0)=0$, this implies that $y \equiv 0$.

Acknowledgments. The authors are partially supported by the french groupement de Recherche (GdR) "Équations d'Amplitudes et Propriétés Qualitatives" (EAPQ), managed by É. Lombardi, of the Centre National de Recherches Scientifiques (CNRS). D. BRESCH is also supported by a RhôneAlpes fellowship obtained in 2004 on problems related to viscous shallow water equations and by the "ACI jeunes chercheurs 2004" du ministère de la Recherche "Études mathématiques de paramétrisations en océanographie".

\section{References}

[AgDoni1] S. Agmon, A. Douglis, L. Nirenberg, Estimates near the boundary for solutions of Elliptic Partial Differential Equations I, Comm. Pure Appl. Math, 12, (1959), 623-727.

[AgDoNi2] S. Agmon, A. Douglis, L. Nirenberg, Estimates near the boundary for solutions of Elliptic Partial Differential Equations II, Comm. Pure Appl. Math, 17, (1964), 35-92. 
[BG] S. Baouendi, C. Goulaouic, Régularité et théorie spectrale pour une classe d'opérateurs elliptiques dégénérés, Arch. Rat. Mech. Anal., 34, (1969), 361-379.

[BC] P. Bolley, J. Camus, Sur une classe d'opérateurs elliptiques dégénérés à plusieurs variables, Mémoires Soc. Math. France, 34, (1973), 55-140.

[BCM] P. Bolley, J. Camus, G. Métivier, Estimations de Schauder et régularité Hölderienne pour une classe de problèmes aux limites singuliers, Comm. Partial Diff. Equ., 11, (1986), 1135-1203.

[BrGiLi] D. Bresch, M. Gisclon, C.K. Lin, An example of low Mach (Froude) number effects for compressible flows with nonconstant density (height) limit. Submitted (2005).

[Ca] C. CAO, Global regularity for certain dissipative hydrodynamical and geophysical systems with an application in control theory. $\mathrm{PhD}$ thesis, Department of Mathematics, University of California, Irvine, (1999).

[Ch] J.-Y. Chemin, Une facette mathématique de la mécanique des fluides. Preprint École Polytechnique, (1993).

[CMR] Th. Clopeau, A. Mikelic, R. Robert, On the vanishing viscosity limit for the 2D incompressible Navier-Stokes equations with the friction type boundary conditions, Nonlinearity, 11, (1998).

[GS] C. Goulaouic, N. Shimakura, Régularité Höldérienne de certains problèmes aux limites dégénérés, Ann. Scuola Norm. Sup. Pisa, 10, (1983), 79-108.

[GS1] C. Goulaouic, N. Shimakura, Régularité Höldérienne de certains problèmes aux limites elliptiques dégénérés, Journées Équations aux dérivées partielles (1981), 1-6.

[Gr] H.P. Greenspan, The theory of rotating fluids, Cambridge University Press, London, (1968).

[LOT] D. Levermore, M. Oliver, E. Titi, Global well-posedness for the lake equation, Physica D, (1996), 492-509.

[Li] P.-L. Lions, Mathematical topics on fluid mechanics, Volume I, Incompressible models, Clarendon Press, Oxford, (1996). 
[Ma] A. MAJDA, Vorticity and the mathematical theory of the incompressible fluid flow. Comm. Pure Appl. Math, 39, (1986), 187-220.

[MaPu] C. Marchioro, M. Pulvirenti, Mathematical theory of incompressible inviscid fluids. Springer-Verlag, New-York (1994).

[BM] A. Majda, A. BertozzI, Vorticity and incompressible flow, Cambdrige Texts in Applied Mathematics, 27. Cambridge University Press, Cambridge, (2002).

[St] E.M. SteIn, Singular integrals and differential properties of functions, Princeton Mathematical Series, 30, Princeton University Press, Princeton, N.J., (1970).

[Yo] V.I. Youdovitch, Nonstationary flos of an ideal incompressible liquid, Zh. Vychisl. Mat. i Mat. Fiz, 6, (1963), 1032-1066. 